\title{
Drivers of Scope of Practice in Family Medicine: A Conceptual Model
}

\author{
Amy Russell, $M D^{1,2}$ \\ Jill Fromewick, $S_{c} D^{2}$ \\ Brittney Macdonald, $M D^{1}$ \\ Stephen Kimmel, $M D^{1}$ \\ Kara Franke, $M P H^{3}$ \\ Kimberly Leach, $\mathrm{MPH}^{2}$ \\ Katby Foley, $\mathrm{PbD}^{2}$ \\ 'HCA Healthcare, Asheville, North \\ Carolina \\ ${ }^{2}$ University of North Carolina (UNC) \\ Health Sciences at MAHEC, Asheville, \\ North Carolina \\ ${ }^{3}$ ToxStrategies, Asheville, North Carolina
}

Conflicts of interest: authors report none.

\section{CORRESPONDING AUTHOR}

Amy Russell

HCA Healthcare

1940 Hendersonville Road

Asheville, NC 28803

amy.russell2@hcahealthcare.com

\begin{abstract}
PURPOSE The purpose of this study was to explore family medicine graduates' attitudes and perspectives on modifiable and unmodifiable factors that influenced their scope of practice and career choices. By understanding how these factors intersect to influence desired and actual scope of practice decisions, we hope to inform strategies to address training and health care workforce needs.
\end{abstract}

METHODS During 5 focus group discussions, comprised of a total of 32 family physicians who either resided in or attended a residency program in western North Carolina, we explored family physicians' attitudes and perspectives on their desired and actual scope of practice. We used thematic analysis to identify patterns in the qualitative data.

RESULTS We created a conceptual framework to understand the complex factors which influence family physicians' scope of practice. Personal factors were found to impact desired scope, while workplace, environmental, and population factors influenced actual scope of practice. Stressors in each of these 4 categories often caused family physicians to narrow their scope of practice. Our study highlights specific supports that, if in place, enable physicians to maintain their desired broad scope of practice.

CONCLUSIONS Our study indicates that the national trend toward family physicians narrowing their scope of practice can be addressed by providing specific supports during training, residency, and mid-career. Understanding personal, workplace, environmental, and population factors that influence scope of practice can inform specific interventions that create desirable jobs for family physicians and improve their ability to meet changing population needs.

Ann Fam Med 2021;19:217-223. https://doi.org/10.1370/afm.2669.

\section{INTRODUCTION}

There is a trend towards an increased scope of practice in the field of family medicine as a whole but individual family physicians' scope of practice has been decreasing over time with fewer family physicians providing basic primary care services, such as hospital, pediatric, and prenatal care. ${ }^{1-3}$ Concurrently, research shows that new family medicine graduates intend to provide a broader scope of practice than current family physicians provide. ${ }^{1,2}$ Reasons hypothesized for the discrepancy between family medicine residents' intentions and the realities of practice are employer constraints and limited practice support. ${ }^{1}$

The value of family physicians providing a broad scope of practice at the physician, patient, and community levels has been reported. Specifically, evidence has suggested that a broader scope of practice and access to comprehensive care is associated with lower rates of physician burnout, greater availability of services, lower hospitalization rates, improved health outcomes, and reduced health care costs. ${ }^{4,5}$

Given the value of a broader scope of practice, researchers have called for further examination of the causes for the decline in the scope of practice. ${ }^{1}$ Reitz and colleagues ${ }^{3}$ recently produced a conceptual model to understand scope of practice decisions for family physicians who originally 
intended to practice rural, full-spectrum medicine. They identified personal, local, and health care system factors which influenced changes from the original desired scope to the eventual actual scope of rural practice.

Our study expands on Reitz's findings and further explores, via focus groups, family medicine graduates' attitudes and perspectives on modifiable and unmodifiable factors that influenced their scope of practice and career choices. By understanding how these factors intersect to influence desired and actual scope of practice decisions, we hope to inform strategies to address training and health care workforce needs.

\section{METHODS}

\section{Study Design}

We employed a qualitative study design using focus groups to gather perspectives from practicing family physicians on factors influencing their scope of practice over time. Focus groups contribute to a rich understanding of a topic through inter-participant dynamics and discussion, and in certain circumstances, data saturation can be reached with a relatively small number of groups. ${ }^{67}$ The Mission Hospital Institutional Review Committee found this study to be exempt.

\section{Setting and Participants}

This study took place in Western North Carolina and included family physicians who worked in or who had trained in the region. We used convenience and purposive sampling to recruit, via e-mail, family physicians in rural and non-rural practice locations, with varying levels of experience post residency to understand how scope of practice changes over time. Five focus groups were conducted with 32 physicians trained in family medicine. We aimed to maintain some homogeneity in experience and rural residence within each group to facilitate discussion. ${ }^{8}$

A total of 5 focus groups were conducted, 2 at our institution, 1 at the rural hospital where focus group participants worked, 1 at a physician's home, and 1 via video conferencing (Zoom Video Communications, Inc) with participants living outside of the region. Participant characteristics were collected via a questionnaire (Table 1).

\section{Data Collection}

The primary investigator (A.R.), a family physician, conducted all focus groups. At least 2 additional coauthors were present at each focus group discussion to observe and take notes and sessions were audio recorded. The same semistructured focus group guide was used for each group. Questions relevant to this article were:
Table 1. Focus Group Participants ( $\mathrm{N}=32$ )

\begin{tabular}{lc}
\hline Characteristics & No. (\%) \\
\hline Physicians & \\
Female & $18(56.3)$ \\
Male & $14(43.8)$ \\
Preceptor & $26(83.9)$ \\
Type of practice & \\
Health system & $6(18.8)$ \\
Teaching group & $6(18.8)$ \\
Community health center & $5(15.6)$ \\
Indian health service & $4(12.5)$ \\
Health system/rural health center & $4(12.5)$ \\
Private group & $4(12.5)$ \\
Solo practice & $1(3.1)$ \\
Academic medical center & $1(3.1)$ \\
Non-clinical public health & $1(3.1)$ \\
Current primary practice location & \\
Rural & $14(43.8)$ \\
Semirural & $2(6.0)$ \\
Urban & $16(50.0)$ \\
State of current practice & \\
North Carolina & $25(78.1)$ \\
Non-NC (CA, Washington DC, KS, & $7(11.9)$ \\
MI, OH, VA, WI) & \\
\hline CA = California; DC = District of Columbia; KS = Kansas; MI = Michigan; \\
NC = North Carolina; OH = Ohio; VA = Virginia; WII = Wisconsin. \\
a Rural status is based on zip code of practice as provided by each focus group \\
participant. Rural designation was defined by Federal Office of Rural Health \\
care and Medicaid Services Rural Health Clinics definition. \\
\hline
\end{tabular}

(1) In what ways is your scope of practice and/or patient population similar to what you expected?

(2) In what ways is your scope of practice and/or patient population different than what you expected?

(3) What factors have determined your scope of practice and/or patient population since residency?

(4) Does the scope of practice and patient population you serve influence your decision to stay in this region or your desire to leave?

\section{Data Analysis}

Audio recordings of the focus group discussions were transcribed by an external transcription firm. Three authors (A.R., J.F., K.F.) reviewed transcripts to generate and agree upon an initial codebook. The lead author (A.R.) and at least 1 additional author coded each transcript in ATLAS.ti 8 (Scientific Software Development $\mathrm{GmbH}$ ) qualitative analysis software, using the initial codebook and adding new codes as they emerged. Five authors (3 aforementioned plus B.M. and S.K.) met regularly to discuss initial and emerging themes, came to a consensus on the relationships between themes, and created a schema to visually depict these relationships. 
Figure 1. Scope of practice of focus group participants $(\mathrm{N}=32)$.

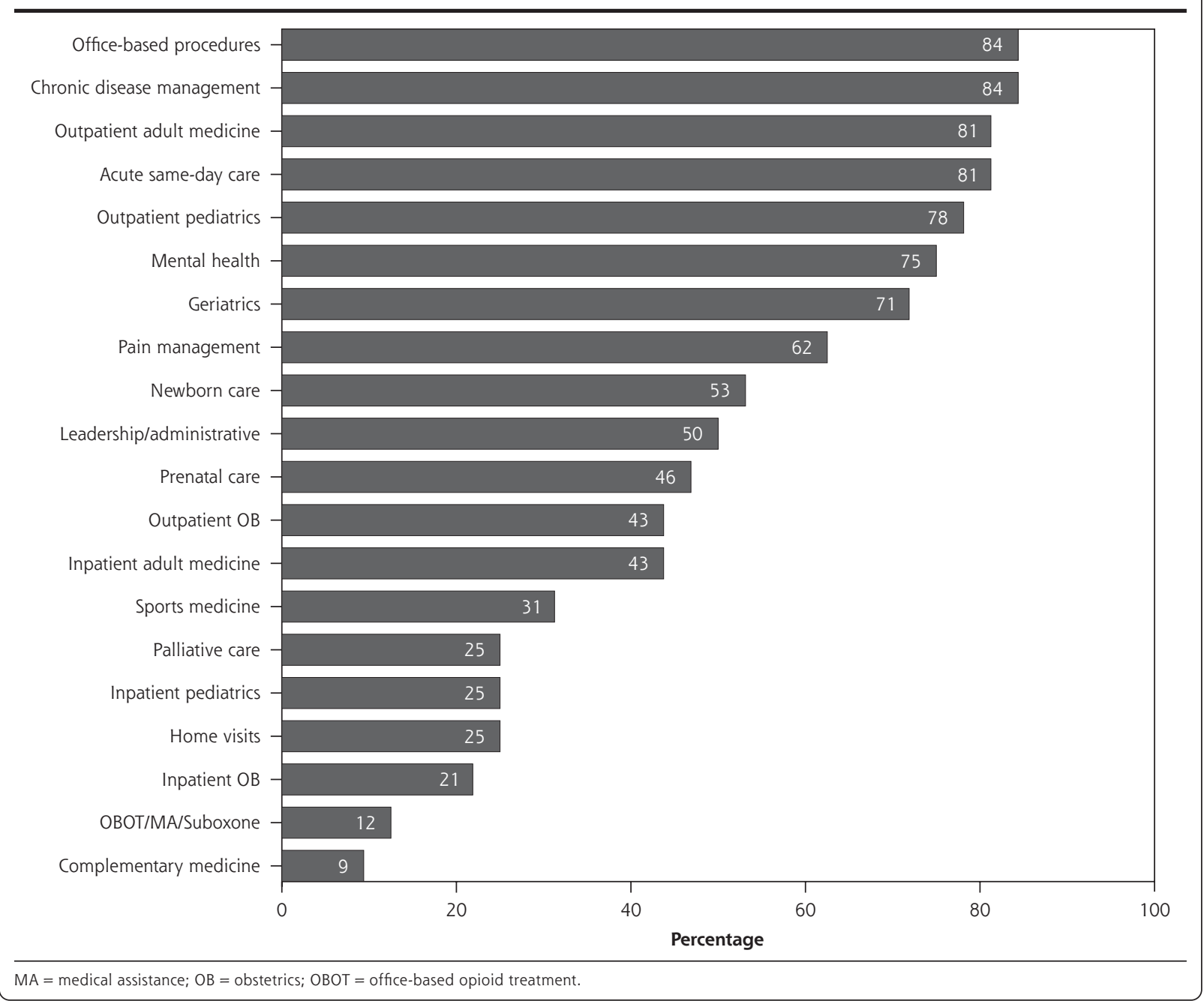

\section{RESULTS}

\section{Participant Characteristics}

Thirty-two family physicians participated in the focus groups (Table 1). Physicians practiced in a wide range of settings and graduated over a span of almost 4 decades, between 1979 and 2017. About 44\% practice primarily in a rural setting, $25 \%$ practice in a small city or town, and about one-third practice in a large city or urban area. Figure 1 illustrates the scope of practice reported by participants. Beyond the options included in the survey, individual participants also reported practicing hospital-based surgeries, abortion care, intellectual and developmental disability care, memory disorders, point-of-care ultrasound, colposcopy, vasectomy, emergency medicine, and public health.

\section{Conceptual Framework}

Through our analysis of the focus group discussions, we identified 4 domains that influence family physicians' future scope of practice: personal, workplace, environment, and population (Figures 2 and 3). Personal factors impacted physician's desired scope of practice, while workplace, environment, and population level factors influenced physician's actual scope of practice. Supports and stressors influenced a physician's decision to narrow their scope, their ability to adapt and maintain scope, or to expand their scope of practice.

\section{Personal Factors}

Consistent with previous studies, most participants highlighted the importance of personal factors in influencing their desired scope of practice. ${ }^{3}$ Each physician brings life circumstances, personal qualities, training, and skills with them to the workplace. Multiple participants highlighted how family circumstances, desired place of residence, and work-life balance caused them to narrow their scope. One physician stated, "[I] always thought I would do rural full scope when I was 
Figure 2. Conceptual model of factors that influence desired and actual scope of practice.

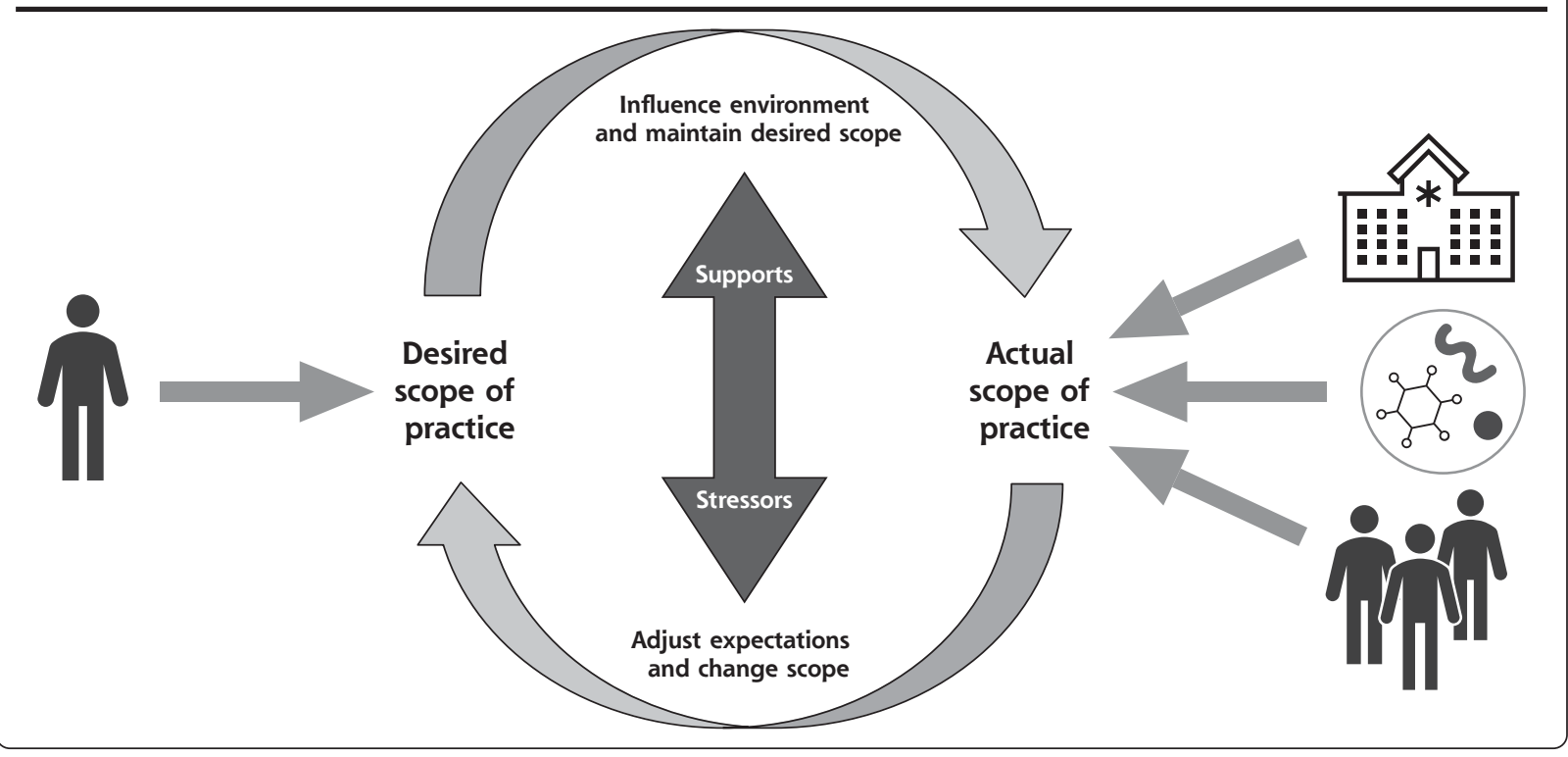

Figure 3. Conceptual model with details of factors that influence desired and actual scope of practice.

\begin{tabular}{|c|c|c|c|c|c|c|c|}
\hline & & & (1) & & & & \\
\hline $\begin{array}{l}\bar{\sigma} \\
\overline{0} \\
\bar{\vdots} \\
\text { a }\end{array}$ & $\begin{array}{l}\text { - Work/life balance } \\
\text { - Personal preference: } \\
\text { population and type } \\
\text { of work } \\
\text { - Family needs } \\
\text { - Desired place to live } \\
\text { - Training } \\
\text { - Mentoring }\end{array}$ & $\begin{array}{l}\stackrel{\Xi}{0} \\
\frac{\pi}{2} \\
\frac{2}{2} \\
3\end{array}$ & $\begin{array}{l}\text { - Work/life balance } \\
\text { - Personal preference: } \\
\text { population and type } \\
\text { of work } \\
\text { - Family needs } \\
\text { - Desired place to live } \\
\text { - Training } \\
\text { - Mentoring }\end{array}$ & 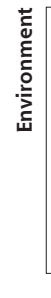 & $\begin{array}{l}\text { - Rural location /isolation } \\
\text { - Local health care economy } \\
\text { - Geography/ proximity to } \\
\text { the hospital } \\
\text { - Local provider mix } \\
\text { - Mental health system } \\
\text { - Health care regulations }\end{array}$ & $\begin{array}{l}\frac{0}{0} \\
\frac{\pi}{3} \\
\frac{0}{0} \\
2\end{array}$ & $\begin{array}{l}\text { - Age demographics } \\
\text { - Social barriers to care } \\
\text { - Population bias towards } \\
\text { specialty care } \\
\text { - Cultural norms regard- } \\
\text { ing approaches to care/ } \\
\text { integrative medicine }\end{array}$ \\
\hline
\end{tabular}

young, and then when I started a family and started a job I'm, like, no!"

Training and resulting skill sets developed during the residency and fellowship years were also identified as a major influence on scope. One participant stated that after focused training in behavioral health, "I was prepared to use my Suboxone license." Others pursued new skills to broaden their scope after residency: "I had to do a lot of fracture management, which I came out of residency clueless about. I got a decent book, learned a local orthopedist I could call, I knew I could bother my colleagues. I figured it out." While some of these personal factors cannot be modified, others were when additional training, mentorship, or support was sought by the physician.

\section{Workplace Factors}

Both employed and independent physicians identified workplace culture, administrative challenges, and staff competencies as either barriers or supports to practicing a broad scope of family medicine. Employed physicians discussed scope of practice limitations set by system administrators and community boards as a result of decisions to silo system services. For example, several physicians lost their ability to practice pediatric medicine when organizational leadership opened a dedicated pediatric clinic. Increased silos also result in challenges finding staff, such as nurses, to support full-spectrum practice: "...in the old hospital the nurses would cross cover inpatient and mother-baby. And now they won't do it anymore. They're just not comfortable doing that."

In some cases, a practice's operational leadership and culture were considered barriers to broad scope practice, "We're allowed to... have kids in our panel, but there's just a lot of institutional resistance to that." Small independent practice physicians reported less resources to address operational challenges and 
implement the staff training needed to manage workflow, quality, and regulatory issues associated with basic primary care services. For example, a physician noted that although they want to be able to serve children with Medicaid, "in my office I don't do vaccines for Medicaid, just because the regulations... so that impacts how much Medicaid pediatrics I do."

In contrast, some physicians reported a work culture that encouraged a broad scope of practice, "[our health system] tradition is built around family doctors doing everything. Even before there was family medicine, there were general practitioners doing everything." Creative workplace solutions sometimes allowed physicians to overcome barriers and practice full scope. For example, one system set up a "command center" so rural physicians, who often feel isolated, could access specialist consultations.

\section{Environmental Factors}

Environmental factors, which we define broadly as the social and physical context that affect individuals and their workplaces ${ }^{9}$ were found to impact physicians' scope of practice. Geography, local provider mix, regional economics, and health care policies were all found to influence scope. In terms of geography, multiple rural physicians narrowed their scope due to travel distances in mountainous terrain to care for patients in hospital and clinic settings, often having to choose between hospital or clinic work.

The mix of clinicians in an area often influenced scope of practice for family physicians. When obstetricians, pediatricians, internal medicine physicians, mental health providers, and specialists are abundant in a community, family physicians are often not called upon to provide a broad scope of services. On the other hand, for several rural physicians, the lack of local specialists resulted in a broadened scope: "There are no specialists who are here, available overnight... so our administration... kind of pushes us in a way to be more broad-spectrum." For both rural and urban physicians, the increasing absence of local mental health and internal medicine clinicians has led them to provide these time-intensive services.

Specifically, in rural areas, factors such as professional isolation and economics shaped scope of practice. In some cases, rural physicians did not have adequate clinical support to sustain all necessary services. As one rural physician stated, "[The community] asked, 'Oh, aren't you going to do OB?' and I said, 'Negative.' I will not be doing that with no surgical back up, no nursing skills, no nothing." In addition, some described how policy and economic factors can impact local services. The closures of local labor and delivery units led to reduced services, and thus, narrowed physicians' scope. "It was the expectation that I would do deliveries in the hospital, but then... it closed one month after I started. So, I didn't get to do any deliveries."

\section{Population Factors}

According to focus group participants, community composition influenced the services they provide. Participants described their efforts to adapt their scope to address changing demographics and community needs. The most common demographic factors known to influence scope were the increase in substance use disorders and the aging population. Many physicians reported that treating the high needs of these populations resulted in less time for lower intensity patients.

The need to care for illnesses disproportionately affecting marginalized populations often led to a broader scope of practice. One physician stated, "When a need exists that nobody else wants to take care of, that opens up our scope." Another acknowledged a colleague: "You were doing HIV care and was one of a very handful of people in all of [our] county that was doing that. And, one of the reasons was because nobody else would do it."

When family physicians take care of low-income populations, patients' lack of insurance may indirectly lead to a broadened scope. When patients have limited access to specialty care, family physicians must adapt and broaden their scope to meet patient needs: "Stuff like that happens all the time. [Specialists are] pretty sure that [family physicians] can't [take care of the patient] until the wallet biopsy comes back negative, and the patient has no insurance and no money, and then they're pretty sure that we can do methotrexate."

Participants also discussed patient and community preferences for specialists and an inadequate understanding of the benefits of comprehensive familycentered care. This bias toward use of specialists reportedly varies based on the cultural and regional background of the patient. One physician noted, "You've got the people who are from the Southeast who are pretty comfortable with seeing a family doctor and then you have the people from the Northeast who pretty much have a different doctor for each organ in their body."

\section{Stressors and Supports}

As shown in the conceptual model (see Figure 2 and Table 2), stressors and supports in each category (personal, workplace, environment, and population) influenced participants' ability to achieve their desired scope. Some of these stressors were not modifiable via intervention, such as family needs (personal), 
productivity expectations (workplace), health care regulations (environment), and demographics of patients (population). Physicians described how unalterable stressors caused them to narrow their scope.

As shown in Table 2, however, some stressors were modifiable when specific supports were present. In spite of pressures that might limit scope, we found that there were supportive factors that led to adaptation and the ability of some family physicians to maintain a broader scope and learn new skills.

Broad-scope training and access to additional medical education after training is essential in preparing physicians to adapt to the needs of their community and to maintain a broad scope of practice. Access to mentors and training supported physicians' adaptation to the stressors associated with isolation. Physicians who maintained a broad scope also reported that organizational leadership support was essential. Organizations who valued full-scope practices created strategies to broaden staff competencies, provided sufficient consultative support, and created more flexible workflows to allow for a wide variety of appointment types. A shift in an organization's culture around scope of practice can occur through the initiatives of strong leaders.

Team-based care including behavioral health, pharmacy, nursing, and nutrition was also identified as a substantial support that helped with population-level stressors such as a shifting need toward the care of complex geriatric and behavioral health populations. Several physicians who participated in rural fellowships described the importance of supported learning in their future rural workplace environment.

\section{DISCUSSION}

Recent literature exploring the narrowing scope of family physicians has focused on personal, local, and health system factors that influence scope of practice. ${ }^{3}$ Our conceptual model adds population factors to the existing understanding of influences on scope of practice. Further, we expanded on local and health system factors described by Reitz and colleagues ${ }^{3}$ to highlight workplace factors that can be influenced by organizational leaders. This research also specifically explores how all these factors apply to family physicians in more rural settings. Because a broad scope of practice is associated with positive outcomes for both family physicians and the community, understanding modifiable and unmodifiable factors that influence scope is essential for targeting interventions at the resident, residency, and employer levels, to ensure that training matches local population and employer needs.

We identified factors in 4 main areas that shape family physicians' scope of practice-personal, workplace, environmental, workplace, and population. Our research confirms prior findings and enhances the understanding of stressors and supports that may modify scope of practice decisions. While stressors in each of the 4 main categories often impact the decision to narrow scope of practice, our study highlights specific supports that, if in place, may enable physicians to maintain a broad scope of practice.

Our interviews indicated that supportive training programs, mentors, and employers can support earlycareer family physicians in maintaining a broader scope of practice. Training programs can also help mid-career family physicians learn new skills. Some supports are more impactful on rural physicians' scope, including availability of trained support staff, local provider mix, local economy, and health system leadership.

Although many participants discussed obstacles to maintaining their scope, the adaptability of those interviewed was evident. With mentorship, additional education, and leadership support, many learned new skills over the years to meet the specific needs of their patient populations. Many physicians interviewed became early adopters of new skills because there was a need in their community. They added, for example, 
care for patients with HIV, hepatitis C, intellectual or developmental disabilities, and opioid use disorder to their scope. Others learned to address transgender care and provided telehealth appointments during the COVID-19 pandemic.

Our study has several limitations. Findings reflect the views and experiences of family physicians located primarily in North Carolina, with a large proportion practicing in rural locations or smaller urban areas. To understand whether the same influences on scope apply in other physicians, future studies should test this conceptual framework in larger urban areas and rural areas not represented in this study. Although the qualitative nature of the study allowed for rich participant perspectives, social desirability is a risk of focus group methodology. In addition, because some participating physicians had been employed in multiple jobs since residency graduation, recall bias may have been an issue. Future studies may also test the relationships in the conceptual framework through surveys and/or prospective methods.

With the national trend indicating a more narrow scope of practice for graduating physicians, leaders have questioned whether a change is needed in family medicine training. ${ }^{10}$ Despite the fact that many participants limited their scope with time, they all acknowledged their gratitude for the breadth of their training which allows them to adapt and be the physician their organization or community needs. Some of the physicians interviewed maintained a broad scope of practice with the support of their organization. We believe that organizations that avoid bias against a broad spectrum of care and understand the factors that influence scope of practice will improve their capability to recruit and retain physicians to serve the specific needs of their population. All communities can benefit from an evidence-based approach to physician workforce planning and the development of support systems to address the personal, workplace, environmental, and population factors that limit physician scope and consequently, patient access to care.
To read or post commentaries in response to this article, go to https://www.AnnFamMed.org/content/19/3/217/tab-e-letters.

Key words: family; rural; scope of practice; workforce

Submitted June 4, 2020; submitted, revised, September 2, 2020; accepted September 28, 2020.

Funding support: This research was unfunded. However, one of the authors, (B.M.) was, at the time the manuscript was drafted, a medical resident in a program partially supported by the Health Resources and Services Administration (HRSA) of the US Department of Health and Human Services (HHS) as part of an award totaling $\$ 1,656,886$ with $0 \%$ financed with non-governmental sources.

Disclaimer: The contents are those of the author(s) and do not necessarily represent the official views of, nor an endorsement, by HRSA, HHS, or the US Government. For more information, please visit HRSA.gov.

\section{References}

1. Coutinho AJ, Cochrane A, Stelter K, Phillips RL Jr, Peterson LE. Comparison of intended scope of practice for family medicine residents with reported scope of practice among practicing family physicians. JAMA. 2015;314(22):2364-2372.

2. Peterson LE, Fang B, Puffer JC, Bazemore AW. Wide gap between preparation and scope of practice of early career family physicians. J Am Board Fam Med. 2018;31(2):181-182.

3. Reitz R, Horst K, Davenport M, Klemmetsen S, Clark M. Factors influencing family physician scope of practice: a grounded theory study. Fam Med. 2018;50(4):269-274.

4. Weidner AKH, Phillips RL Jr, Fang B, Peterson LE. Burnout and scope of practice in new family physicians. Ann Fam Med. 2018; 16(3):200-205.

5. Bazemore A, Petterson S, Peterson LE, Phillips RL Jr. More comprehensive care among family physicians is associated with lower costs and fewer hospitalizations. Ann Fam Med. 2015;13(3):206-213.

6. Rauf A, Baig L, Jaffery T, Shafi R. Exploring the trustworthiness and reliability of focus groups for obtaining useful feedback for evaluation of academic programs. Educ Health (Abingdon). 2014;27(1): 28-33.

7. Guest G, Namey E, McKenna K. How many focus groups are enough? Building an evidence base for nonprobability sample sizes. Field Methods. 2017;29(1):3-22.

8. Kreuger RA, Casey MA. Focus Groups: A Practical Guide for Applied Research. SAGE Publications; 2009.

9. Woolf $\mathrm{SH}$, Aron L, eds. US Health in International Perspective: Shorter Lives, Poorer Health. National Academies Press; 2013.

10. Spogen D. Shrinking scope of practice raises questions about future of family medicine training. Published Mar 22, 2012. Accessed Aug 27, 2018. https://www.aafp.org/news/blogs/leadervoices/entry/ shrinking_scope_of_practice_raises.html 